\title{
A HYBRID MODEL FOR THE EVALUATION OF END USER EXPERIENCE IN A BRING YOUR OWN DEVICE ENVIRONMENT USING MULTIFACTOR AUTHENTICATION
}

\author{
Annie Hennelly, Sharon Gurry, Kieran Flanagan and Enda Fallon \\ Athlone Institute of Technology, Co. Westmeath, Ireland
}

\begin{abstract}
There is a growing trend of Bring Your Own Device (BYOD) adoption in companies; the controls such as policies, companies attitude to risk, technological solutions that offer an additional layer of security help companies to protect themselves against risks such as data leakage or social engineering. We propose that companies focus is absolutely warranted to ensure the confidentiality and privacy of its assets but user experience must not be forgotten with this rising trend. In this paper we took an existing model Gupta (2018) used to measure user's intention to adopt BYOD and modified and utilised it to further investigate User Experience (UE) in relation to Multifactor Authentication (MFA) and BYOD. We implemented the model in a similar fashion as Gupta's study and created a number of hypothesis to evaluate the user's response and determine their experience to be positive, negative or neutral. The results indicated that there was not a correlation between perceived ease of use of MFA and perceived usefulness or user experience. While surprising this too was observed by Gupta and is an area further study is required. There were strong correlations observed however in positive perceived ease of use and personal innovativeness on users' positive attitude towards MFA which in turn could likely impact user experience.
\end{abstract}

\section{KEYWORDS}

Bring Your Own Device (BYOD), Multifactor Authentication (MFA), User Experience

\section{INTRODUCTION}

Although organisations invest in training their employees in Cyber Security awareness there is always the risk that human error will prevail as more companies are opting to allow employees to BYOD. The end-user experience is often forgotten when implementing security technologies in an organisation. It is critical to the success of an organisation to have both a secure environment coupled with a seamless employee experience.

Implementing multifactor authentication as part of an organisations security strategies and policies while not diluting the end-user experience allows the organisation to add an additional and critical layer of security while maintaining employee satisfaction by enabling ease of doing business.

Using the following criteria we selected a subset of papers that met our inclusion/exclusion criteria for this study.

- $\quad$ Representative of interests

- $\quad$ Shared purpose

- $\quad$ Appropriateness of the methods/approach - Enterprise

- Multifactor/BYOD related 


\section{LITERATURE REVIEW}

\subsection{Introduction}

This literature review explores the growing trend of Bring Your Own Device adoption in companies; the controls such as policies, attitude to risk \& technological solutions that offer an additional layer of security to help companies to protect themselves. We propose that companies while security focus is absolutely warranted to ensure the confidentiality and privacy of its assets the user experience must not be forgotten with the rising trend of (BYOD).

BYOD advantages as described by Tanimoto (2016) are increased productivity and opportunities for new business and services. He also discusses risks with BYOD adoptions such as information leakages; company's confidential data or an employee's personal information.

A popular measure to securing mobile devices has been with the use of multifactor authentication. These controls while imperative to the security posture of the organisation should also work without not diluting the end-user experience. We propose that it is critical to the success of an organisation to have both a secure environment but coupled with a seamless employee experience.

\subsection{MFA Bring Your Own Device (BYOD)}

As discussed by Oktavia (2016) there are implications BYOD policies bring to the security and privacy of the organisation. Privacy in this context is defined as the behaviour of the organisation to protect their information assets and their customer personally identifiable information. As also observed in other research (Tanimoto et al., 2016) advantages such as employee satisfaction and heightened employee productivity are key advantages of adopting BYOD. This paper recognizes the risk in lowering the control of an organisation by giving the employees the responsibility to protect the organisations.

The critical components according to this study into BYOD view the main challenges to be privacy and security related. This research advocates for the implementation of adequate BYOD policies as well segregation of the corporate space from personal data with the goal of protecting corporate data (Oktavia et al., 2016).

Tanimoto (2016) proposes a risk assessment strategy which reviews operations and systems and then applies the appropriate risk strategy; transference, avoidance, mitigation. The study concluded that information leakage was the biggest risk and that a mobile device management system was the strongest countermeasure (Tanimoto et al., 2016).

One of the few papers we found that examined BYOD from the perspective of the employee is by Gupta (2019) which looks to evaluate the perception of the employees to BYOD and their intention to adopt their personal devices for organisational purpose. This paper's proposed model suggests the relationship amongst Personal Innovativeness (PI), Perceived Ease of Use, Attitude (ATT), Perceived Usefulness (PU) and Intention to adopt (INTADOPT). In this model, various constructs namely- Personal Innovativeness (PI), Perceived Ease of Use (PEOU), Perceived Usefulness (PU) \& Attitude have been utilized to analyse the adoption intention (INTADOPT). PEOU and PU proved to be important factors that impact attitude, while personal innovativeness was found to have insignificant impact on attitude. Likewise, attitude and personal innovativeness significantly affect the employees' intention to adopt BYOD whereas PEOU seems to have no impact on adoption intention. So it findings implied that ease of use is not a major factor that could attract the employees to opt for BYOD.

As with other papers reviewed user experience is omitted from considerations, the focus is either on the technology, policy, the risks and the controls required to mitigate the risk (Seneviratne et al., 2018; Herrera, 2017; Gupta, 2018). It did however emphasize the need to consider the users emotional reactions to BYOD policies in relation to confidentiality and security considerations. The finding that ease of use was not a prominent factor in BYOD adoption is very interesting and requires further study (Gupta et al., 2019). 


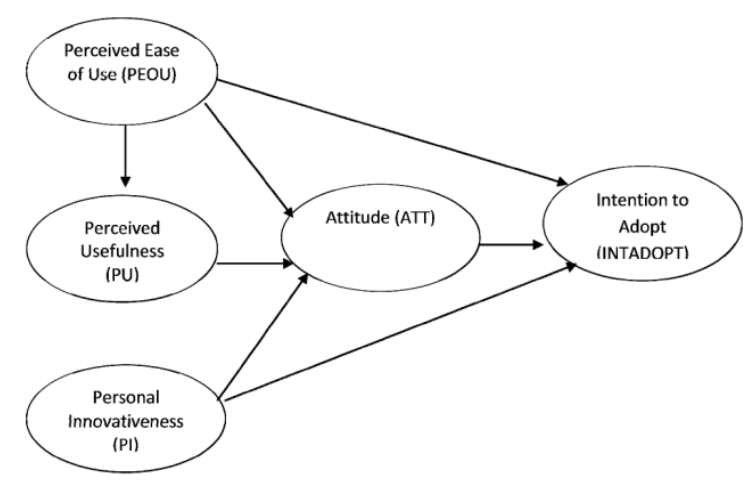

Figure 1. Gupta Model (Seneviratne et al., 2018)

\subsection{MFA in Relation to User Experience}

Due to increase in security incidents and the scale of data breaches in recent years, users have been made more aware of authentication and multifactor authentication (MFA). MFA is generally something you have (device), something you know (password) and something you are (biometrics). From a user experience standpoint having an easy way to access your own accounts is vital while still having that additional layer of security protecting the underlying data and information. While users may find some authentication methods a hindrance, they also need to consider the amount of personal data stored on their device with the increasing usage of phone applications supporting everyday activities such as email and banking. (Bissada Andrew, 2017).

The introduction of BYOD means that we must look at MFA as a service that meets the user experience demands of the users. Seto (2015) suggests from previous studies users prefer usability over suitability however by this notion alone, the user is introducing risk to their device. The authors suggest that by introducing user habit oriented authentication along with traditional authentication creates a more user friendly experience. (Yan, 2018). It could be concluded from this article that there is a need for a mind-set change when it comes to authentication types for the end user.

An interesting aspect of user experience is the relationship between quality of service (QoS), quality of experience (QoE) and quality of protection (QoP). Because there are very few user-friendly and secure authentication services available it is difficult to find the correct balance of usability and security. If the authentication method is easier to use it will drive better quality of service meaning users will have a better experience and in turn will increase security on their device (Sepczuk, 2016).

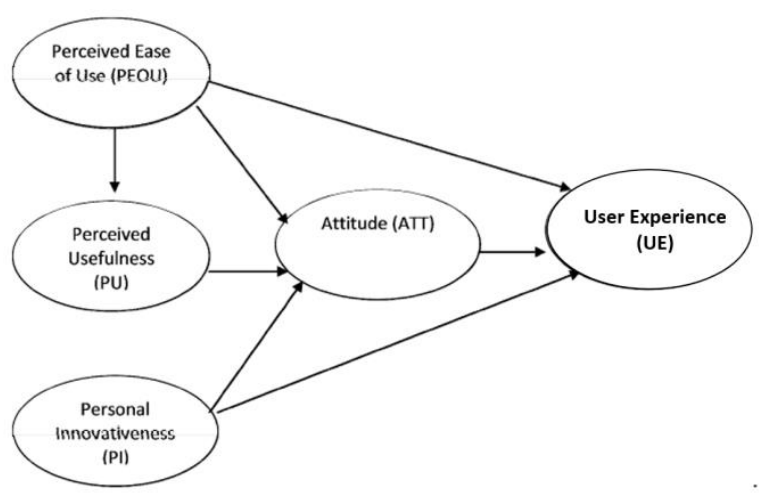

Figure 2. Proposed Model 


\section{RESEARCH APPROACH AND RESULTS}

As previously outlined a study was conducted by Gupta (2019) into users perception to adopt BYOD conducted using a proposed model [Figure 1], a hybrid between TAM (Technology Acceptance Model) model and the TRA (Theory of Reasons Action) model. We propose to modify this model and utilise it to further investigate User Experience (UE) into MFA and BYOD via a questionnaire.

We proposed to achieve this by taking the main components of the model which were relevant and replace "Intention to Adopt" with a new factor "User Experience" (UE). We will apply the model in a similar fashion to Gupta and have developed a number of hypothesis that will help us evaluate the user's response and determine their experience to be positive, negative or neutral to each of the factors thus deriving a result to the relevant hypothesis.

\subsection{Hypothesis Definition \& Path}

Table 1. Hypothesis Definition \& Path

\begin{tabular}{|c|c|c|}
\hline Hypotheses & $\begin{array}{l}\quad \text { Description } \\
\text { Perceived Ease of Use (PEOU) has a positive effect on Perceived } \\
\text { Usefulness (PU) }\end{array}$ & PEOU $>$ PU $>$ ATT $>$ UE \\
\hline $\mathrm{H} 2$ & $\begin{array}{l}\text { Perceived Ease of Use (PEOU) has a positive effect on Attitude } \\
\text { (ATT) }\end{array}$ & PEOU $>$ ATT $>$ UE \\
\hline $\mathrm{H3}$ & $\begin{array}{l}\text { Perceived Ease of Use (PEOU) has a positive effect on User } \\
\text { Experience (UE) }\end{array}$ & PEOU $>\mathrm{UE}$ \\
\hline $\mathrm{H} 4$ & $\begin{array}{l}\text { Perceived Usefulness (PU) has a positive effect on Attitude (ATT) } \\
\text { which can in tum have a positive effect on User Experience (UE) }\end{array}$ & $\mathrm{PU}>\mathrm{ATT} \gg \mathrm{UE}$ \\
\hline H5 & $\begin{array}{l}\text { Attitude (ATT) has a positive effect on Personal Innovativeness } \\
\text { (PI) which can in turm have a positive effect on User Experience } \\
\text { (UE) }\end{array}$ & PI $>$ ATT $\gg$ UE \\
\hline $\mathrm{H} 6$ & $\begin{array}{l}\text { Personal Innovativeness }(\mathrm{PI}) \text { has a positive effect on User } \\
\text { Experience }(\mathrm{UE})\end{array}$ & $\mathrm{PI} \gg \mathrm{UE}$ \\
\hline
\end{tabular}

The goal is to take security out of the equation to allow the focus to be on the user and their experience. The aim is to get to the root cause of any trending issues that users may experience when multifactor authentication measures are implemented. The responses of the survey will allow conclusions to be drawn around the end user experience which could prompt organisations to take tangible action to allow for a better user experience while still promoting and retaining security.

A subset of the survey questions used to derive either a positive, negative or neutral response to each of the hypothesis include:

Perceived Ease of Use (PEOU)

- In general, MFA technologies are easy to use? 1= Strongly Agree, 2=Agree, 3=No Opinion, 4=Disagree, 5=Strongly Disagree

Perceived Usefulness (PU)

- If you had the option to opt out of MFA would you? Yes/No

- You understand the need for and the importance of MFA? Strongly Agree, 2=Agree, 3=No Opinion, 4=Disagree, 5=Strongly Disagree

Personal Innovativeness (PI)

- If your organisation allowed you to BYOD would you want MFA to be part of the policy? Yes/No

- If your organisation allowed you to BYOD would you want to use your own personal device? Yes/No Attitude (ATT)

- In general my experience to date using MFA has been positive? Strongly Agree, 2=Agree, 3=No Opinion, 4=Disagree, 5=Strongly Disagree

- How would you feel if your personal data was leaked? 1=Angry, 2=Neutral, 3= Don't care 
There are a number of MFA solutions and implementations available on the market today that support BYOD. The MFA technologies considered for the scope of the survey and analysis include:

1. Microsoft Multifactor Authentication

2. PING ID

3. Okta

4. Google Authenticator

5. Amazon Web Services

6. DUO

\section{REVIEW OF MFA TECHNOLOGIES}

\subsection{Google Authenticator}

Google Authenticator is a two-step verification application that uses a one-time password to authenticate users in addition to their normal log on details (Wikipedia, 2020). Once a user has set up two-step verification they will be required to enter in their log on password however they will be prompted for a second verification to prove their identity. A user can get a one-time password via a text, voice call or through the google authentication app on their smartphone. When the user signs in they will be given an option to save that verification on that device meaning that the user will still have that two-step verification on that computer (Google, 2020). While this seems convenient for the user, it could prove to be a dangerous feature if the user selects this option on a publicly used computer such as in an internet café or library. While there is an option to integrate single sign on to the Google two-step verification process, Google themselves do not offer this feature rather third party suppliers offer this integration adding another layer of complexity into the mix.

\subsection{Amazon Web Services (AWS)}

According to Amazon Web Services AWS MFA adds an additional layer of security on top of the usual password and user name. When using AWS the user signs on to an AWS console they are prompted for their username and password followed up with the authentication code from their chosen additional device (Amazon Web Services, 2020).

While it seems that AWS SSO and AWS MFA are sold as separate services by Amazon Web Services, it is up to the customer how they want their users to authenticate. There is the option to have full single sign on plus MFA where the user will only be prompted if signing in from what appears to be an untrusted device. Another option a customer could impose on their users is that MFA is required for every sign on (Amazon Web Services, 2020).

\subsection{DUO}

DUO have designed their MFA on the basis of a zero trust approach. Because there are more remote workers and BYOD policies, employees now expect that the workplace will give them the freedom to choose their devices. DUO suggests that by using their products they can address potential security concerns by creating trust for every access request regardless of where they are located. DUO operates like any other two-factor authentication product by having something you know and a physical device. It claims to be simple and user friendly while integrating with the existing technologies that the business is using. The company offers single sign on as a separate service however it is not clear if this is a default feature that comes with the product (DUO, 2020). 


\subsection{Microsoft Multifactor Authentication}

Microsoft(MS) has been named a leader in the Garner Magic Quadrant for Access Management. Their proclaimed goal is to ensure that all organizations have a basic level of security enabled at no extra cost. The setup of MS MFA is straight forward and not unlike the other providers, you can become MFA enabled for MS products with a click of a button. There are a number of alternatives for the end user to interact with MS MFA solution: call, text to mobile or office phone, notification and one time code via Microsoft authentication app and passwordless sign-in using biometrics. At an Enterprise level MS has a list of 9 privileged accounts that it proposes must be treated with "special care". This equates to requesting the users who are tied to these accounts to perform additional authentication every time they sign in. this is very clunky and is a security only view of the use case (Microsoft, 2020).

\subsection{Okta}

Okta claim to offer a "complete access management platform, securing critical resources from the cloud to the ground" and have been named as leaders in the Gartner Magic Quadrant for access management for the third consecutive year. Okta implement a zero trust approach to security and have a portfolio of Access Management solutions from Universal Directory - a flexible cloud based user store to customize, organize, and manage any set of user attributes, LifeCycle Management - which automates user onboarding and offboarding, Adaptive MFA and Single Sign-On, a single set of credentials gives your people access to all apps across devices. Okta boast an enhanced user and admin experience with their MFA offering authentication without a password, seamless enrolment, flexible authentication offering various end user experiences including 1-click authentication, simple reporting and auditing such as authentication logs i.e. login attempts (Okta, 2020).

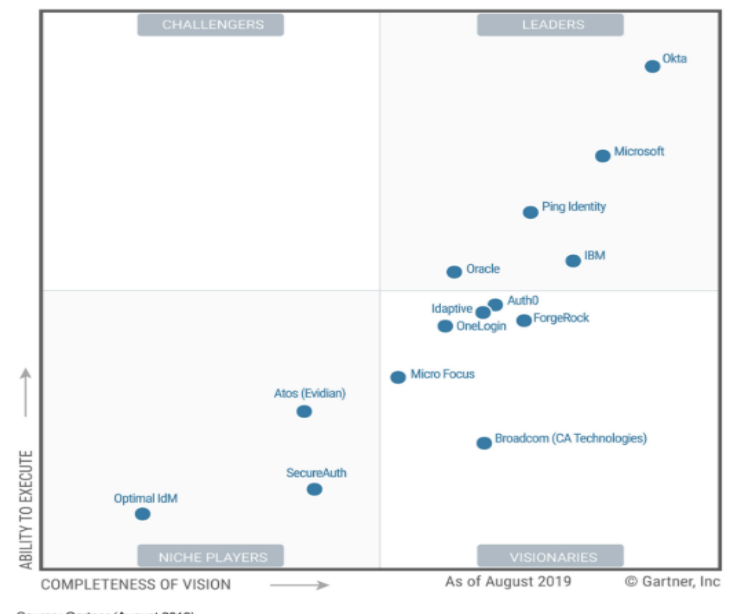

Figure 3. Magic Quadrant for Access Management

\subsection{Ping Identity}

Ping ID has also been named a leader in the Garner Magic Qudrant for Access Management. PingID is a cloud based MFA solution that is used in Enterprise. It's capability includes protecting workforce apps accessed via web single sign-on (SSO), integrations with Microsoft Azure AD, o365, Active Directory Federation Services (AD FS) and Windows Login thus supporting a hybrid IT environments that utilize a mix of on-premises, private cloud and SaaS applications. Ping ID positions itself to provide balance of security with convenience which is very much in line with what we are advocating for in this paper. When policy dictates the need for strong authentication, the PingID service will send a notification to the user's registered device through the PingID mobile app to swipe for authentication. This too supports biometric authentication for the ultimate user convenience (PingIdentity, 2020). 
On reflection of the technologies and findings we decided to reduce the number of technologies in scope for evaluation and focus on Microsoft Multifactor Authentication and Ping Identity. This presents an opportunity for future study with the inclusion of Okta, the remaining leader per Gartner 2019 ratings.

\section{RESULTS}

For the purposes of anonymity we will refer to the technologies as T1 and T2 when we discuss results. Per the analysis of the results the overall determination of the hypothesis in scope for this study are as follows:

Table 2. Hypothesis Results

\begin{tabular}{|clc|}
\hline Hypotheses & \multicolumn{1}{c|}{$\begin{array}{c}\text { Description } \\
\text { Perceived Ease of Use (PEOU) has a positive effect on Perceived }\end{array}$} & Nosult \\
\hline Usefulness (PU) & Yes \\
\hline H2 & $\begin{array}{l}\text { Perceived Ease of Use (PEOU) has a positive effect on Attitude } \\
\text { (ATT) }\end{array}$ & No \\
\hline H3 & $\begin{array}{l}\text { Perceived Ease of Use (PEOU) has a positive effect on User } \\
\text { Experience (UE) }\end{array}$ & Yes \\
\hline H4 & $\begin{array}{l}\text { Perceived Usefulness (PU) has a positive effect on Attitude (ATT) } \\
\text { which can in turn have a positive effect on User Experience (UE) }\end{array}$ & No \\
\hline H5 & $\begin{array}{l}\text { Attitude (ATT) has a positive effect on Personal Innovativeness } \\
\text { (PI)) which can in turn have a positive effect on User Experience } \\
\text { (UE) }\end{array}$ & No \\
\hline H6 & $\begin{array}{l}\text { Personal Innovativeness (PI) has a positive effect on User } \\
\text { Experience (UE) }\end{array}$
\end{tabular}

Key findings were as follows:

- In general respondents found authentication technologies easy to use and were open to using another authentication method if the current solution was not in place.

- H1: There is a correlation between the Perceived Ease of Use (PEOU) and the number users are required to authenticate per day. However given one technology rated much higher in Perceived Ease of Use it did not seem to impact the results overall for Perceived Usefulness (PU) [Figure 4].

- H2: In general Perceived Ease of Use has an impact on Attitude (ATT). There is a strong correlation between the technology and the results for Perceived Ease of Use with attitude scoring significantly higher for the technology that was easier for the users' to interact with [Figure 5].

- H3: There isn't an obvious correlation between Perceived Ease of Use and User Experience (UE) in a positive or negative way. The findings indicate the overriding understanding of the need for MFA and the implications of a security breach was higher in priority to the sample surveyed who had a high level understand of IT and Security practices [Figure 6].

- H4: Perceived Usefulness (PU) findings suggest a strong correlation between the perceived value of MFA and users Attitude (ATT). This is tied to users understanding of the importance of MFA. Majority of respondents felt their data was more secure with MFA and would not opt out if given the opportunity [Figure 7].

- H5: The results here were more divisive in relation to Personal Innovativeness (PI). Vast majority of the sample would want MFA to be included as part of a BYOD corporate policy and felt they would be equipped to secure corporate data. However, there was almost a 50:50 split between the desire to use one's own personal device. It is the determination from the results that the overarching positive response in Attitude (ATT) towards MFA would influence Personal Innovativeness (PI) but not necessarily User Experience (UE) [Figure 8].

- H6: The results did not support this hypothesis. Users may see the value of MFA and BYOD but there was a reluctance to use a personal device for corporate activities. Users demonstrated great concern for breach which may impact both corporate and sensitive data [Figure 9]. 
In general, MFA technologies are easy to use? \& \# of Authentication Per Day Ease: $1=$ Strongly Agree, $2=$ Agree, $3=$ No Opinion, 4=Disagree, $5=$ Strongly Disagree Authentication Times: $1=$ less than one, $2=$ less than five, $3=$ more than five

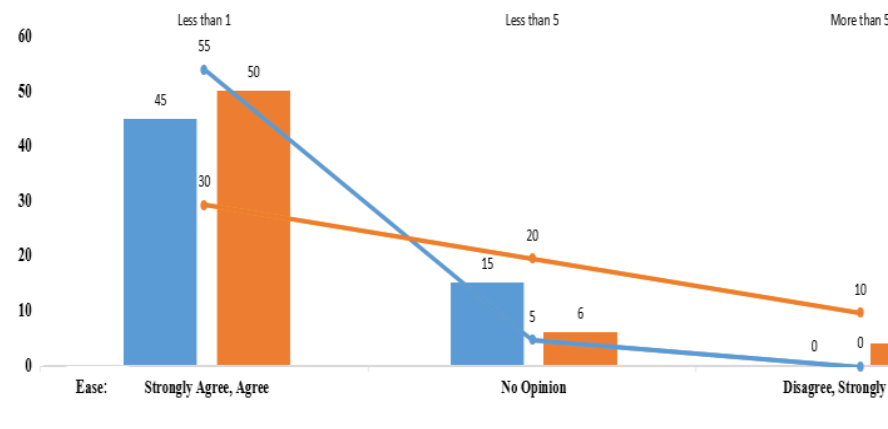

$\because \mathrm{T} 1 \quad$ "T2
Do you see the value in using MFA?

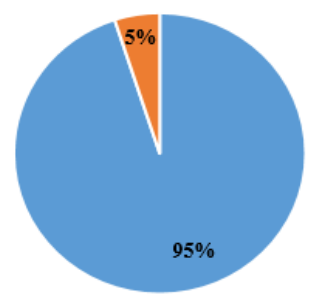

- Yes $=$ No
In general, my experience to date using MFA has been positive? $1=$ Strongly Agree, $2=A g r e e$, $3=$ No opinion,, 4=Disagree, $5=$ Strongly Disagree \&and Average of How many clicks does it take to get to the appicaiton you want to use when MFA is enabled?

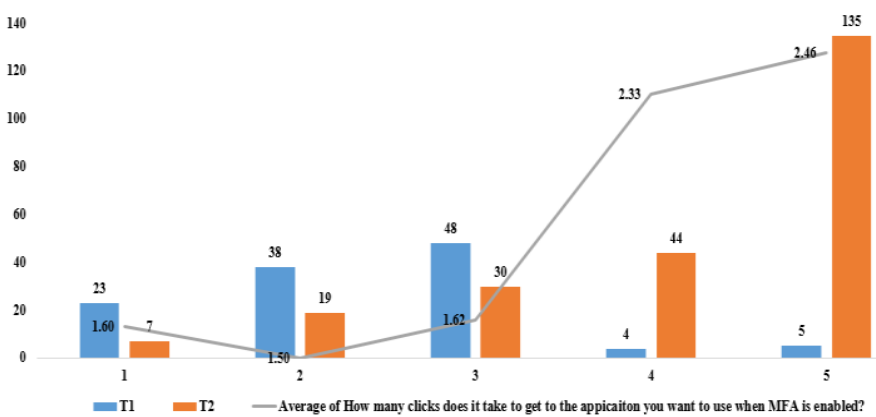

Do you understand the need for MFA? Yes/No

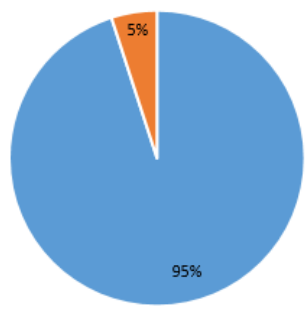

"Yes $=$ No

In general, MFA techologies are easy to use? and Average of How many clicks does it take to get to the appicaiton you want to use when MFA is enabled?

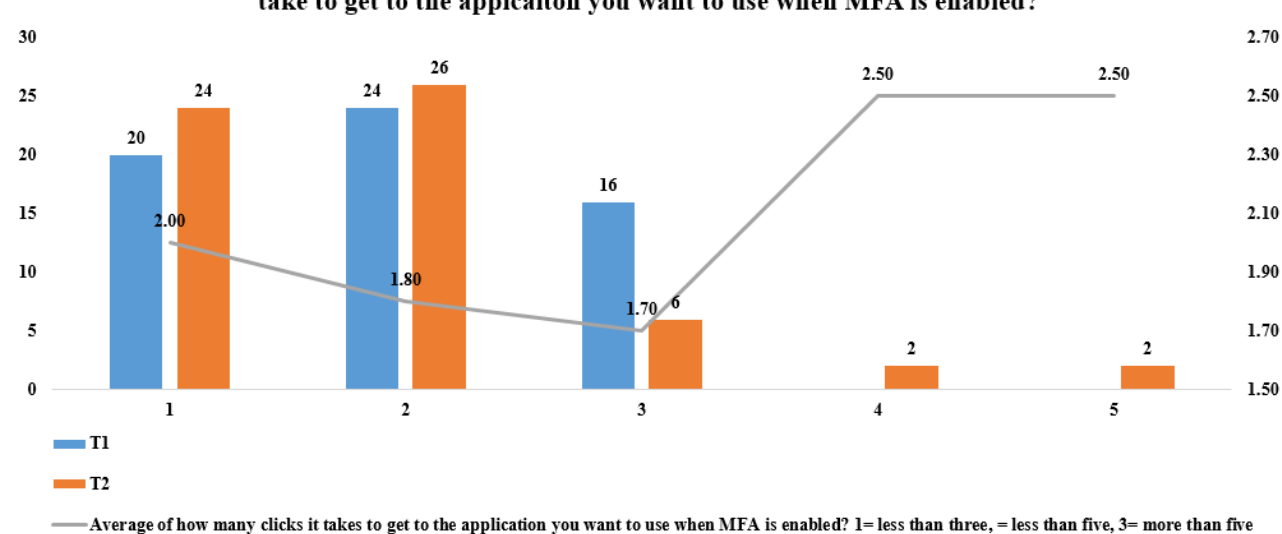



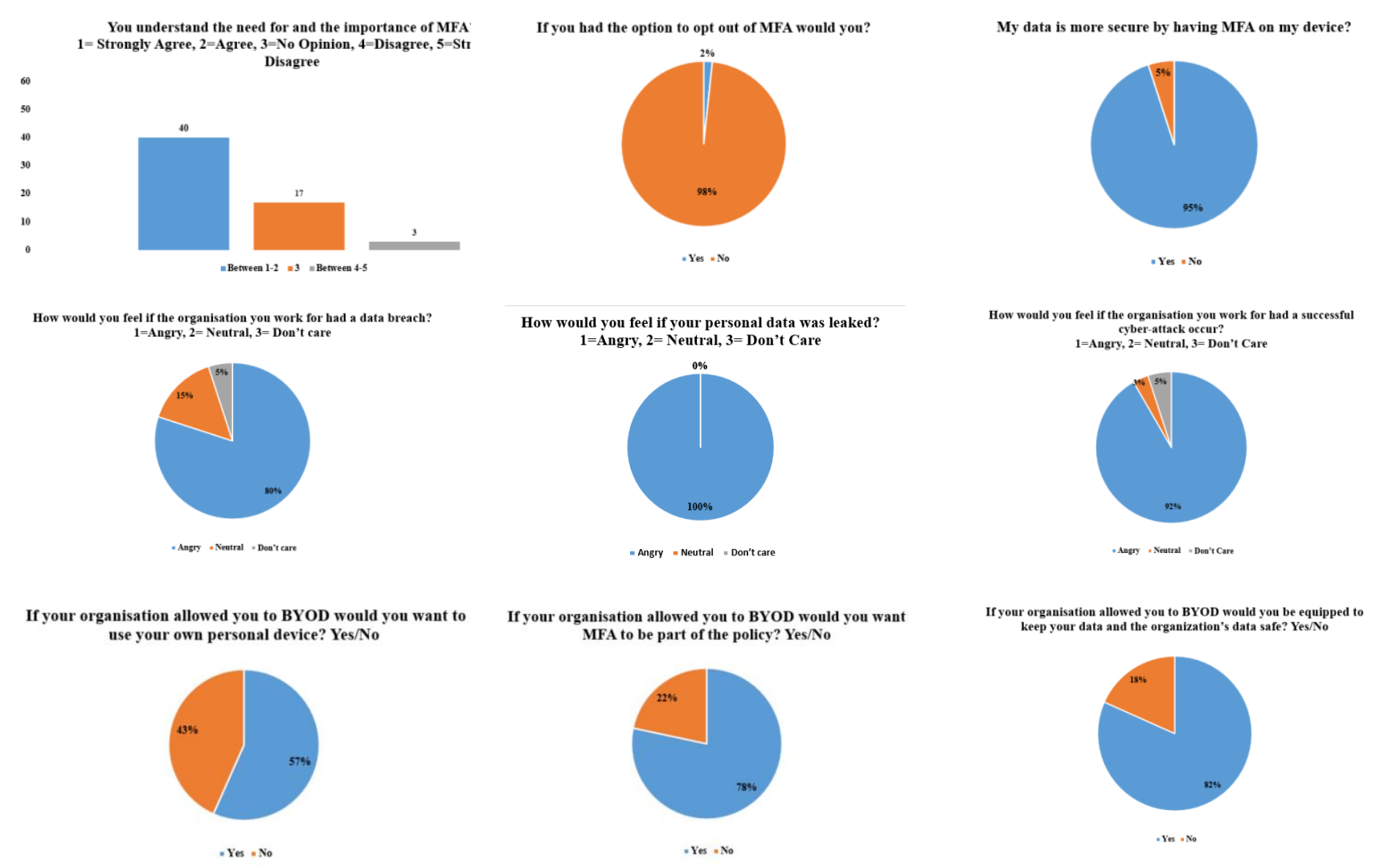

Figure 4. An Analysis of the Perceived Ease of Use and Applicability of MFA Technologies

\section{CONCLUSION}

Per the research review data leakage, confidentiality and privacy implications are considered to be at the higher spectrum of the associated risks for BYOD. Risk analysis, comprehensive security policies and technological countermeasures such as MFA and MDM (Mobile Device Management) are advocated as being a solution to the challenge posed to the organisation. But what does this mean for the end user? It appears that user experience generally not a consideration even when there are controls implemented on them and the blurring of lines between private and professional with BYOD, but does it really matter?

While a suitable model was found to understand user's willingness to adopt BYOD it lacked the perspective of user satisfaction. For the purposes of this study the model was modified to also include User Experience (UE) as well as end users Perceived Ease of Use (PEOU), Perceived Usefulness (PU), Personal Innovativeness (PI), Attitude (ATT) and towards MFA and MFA in relation to BYOD.

As with the original model there were some surprising results with regards to user experience. The results were contrary to the belief this paper set out that user experience as perceived by users should be an equal consideration to technological and security controls imposed on employees with MFA and BYOD. Perceived Usefulness (PU) and Attitude (ATT) as well as an understanding of the need for secure devices and protection of personal and corporate data was a higher priority for the sample than User Experience. This is highly likely due to the sample having security or IT background. This was demonstrated through the results of $\mathrm{H} 1, \mathrm{H} 3, \mathrm{H} 5$ \& H6. Another interesting finding is in relation to BYOD in H5 \& H6 where almost 50:50 divide in the willingness to use a personal device and less satisfaction for tools such as MFA to be installed on a personal device for corporate use even though the value of the tools are understood. This is understandable as with BYOD users relinquish an element of control and privacy with their own device. The survey sample were skilled IT and security professionals which likely influenced the outcome towards to practical versus experience. This leaves the opportunity for further study in this area with a sample from a non-technology/security background as well as the inclusion of additional MFA technologies. 


\section{REFERENCES}

Amazon Web Services. (2020). Enable Multi-Factor Authentication. Docs.aws.amazon.com. Retrieved from https://docs.aws.amazon.com/singlesignon/latest/userguide/enable-mfa.html.

Amazon Web Services. (2020). Multi-factor Authentication. Amazon Web Services, Inc. Retrieved from https://aws.amazon.com/iam/features/mfa/?audit=2019q1.

Bissada Andrew, O. (2017). Mobile Multi-Factor Authentication. In The 12th International Conference for Internet Technology and Secured Transactions (pp. 210-211). ICITST-2017.

Dostálek, L. (2019). Multi-Factor Authentication Modeling. In 9th International Conference on Advanced Computer Information Technologies (pp. 443-446). ACIT.

DUO. (2020). Two-Factor Authentication - 2FA. Duo Security. Retrieved 29 February 2020, from https://duo.com/product/multi-factor-authentication-mfa/two-factor-authentication2fa?utm_medium=paid_search\&utm_source=google\&utm_campaign=EMEA_Brand\#eyJoYXNoIjoiliwic2VhcmNoI joiP3V0bV9tZWRpdW09cGFpZF9zZWFyY2gmdXRtX3NvdXJjZT1nb29nbGUmdXRtX2NhbXBhaWduPUVN.

Google. (2020). Google 2-Step Verification. Google.com. Retrieved from https://www.google.com/landing/2step/\#tab=why-you-need-it.

Gupta, R., Bhardwaj, G., \& Singh, G. (2019). Employee Perception and Behavioral Intention to Adopt BYOD in the Organizations. In International Conference on Automation, Computational and Technology Management (pp. 73-78). London, United Kingdom; ICACTM.

Gupta, S. (2018). Single Sign-On beyond Corporate Boundaries. In 8th International Conference on Intelligent Systems, Modelling and Simulation (pp. 38-42). ISMS.

Herrera, A., Ron, M., \& Rabadão, C. (2017). National cyber-security policies oriented to BYOD (bring your own device): Systematic review. In 12th Iberian Conference on Information Systems and Technologies (pp. 1-4). CISTI.

Lee, K., \& Kim, S. (2020). A security architecture for BYOD office. In International Conference on Advanced Technologies for Communications (pp. 487-490). ATC.

Microsoft. (2020). Multi-factor authentication (MFA) - Microsoft Security. Microsoft.com. Retrieved from https://www.microsoft.com/en-us/security/business/identity/mfa.

Ogie, R. (2016). Bring Your Own Device: An overview of risk assessment. IEEE Consumer Electronics Magazine, 114-119.

Okta. (2020). Multi-factor Authentication (MFA) Solution. Okta.com. Retrieved from https://www.okta.com/products/adaptive-multi-factor-authentication.

Oktavia, T., Yanti, Prabowo, H., \& Meyliana. (2016). Security and privacy challenge in Bring Your Own Device environment: A Systematic Literature Review. In 2016 International Conference on Information Management and Technology (pp. 194-199). Bandung; ICIMTech.

PingIdentity. (2020). Multi Factor Authentication Solutions, MFA Authentication. Pingidentity.com. Retrieved from https://www.pingidentity.com/en/platform/multi-factor-authentication.html.

Seneviratne, B., \& Senaratne, S. (2018). Integrated Corporate Network Service Architecture for Bring Your Own Device (BYOD) Policy. In 2018 3rd International Conference on Information Technology Research (pp. 1-6). ICITR.

Sepczuk M, K. (2018). A new authentication management model oriented on user's experience. In Federated Conference on Computer Science and Information Systems (pp. 1021-1030).

Seto, J., Wang, Y., \& Lin, X. (2015). User-Habit-Oriented Authentication Model: Toward Secure, User-Friendly Authentication for Mobile Devices (pp. 107-118). EMERGING TOPICS.

Tanimoto, S., Yamada, S., Iwashita, M., \& Kobayashi, T. (2016). Risk assessment of BYOD: Bring your own device. IEEE Consumer Electronics Magazine, 114-119.

Wikipedia. (2020). Google Authenticator. $\quad$ En.wikipedia.org. Retrieved from https://en.wikipedia.org/wiki/Google_Authenticator.

Yan, J., Qi, Y., Rao, Q., \& Qi, S. (2018). Towards A User-friendly and Secure Hand Shaking Authentication for Smartphones. In 17th IEEE International Conference On Trust, Security And Privacy In Computing And Communications (pp. 1170-1179). IEEE. 